\title{
Sur Jean Kaempfer, et sur Proust (à propos des pastiches)
}

\section{Franc Schuerewegen}

\section{(2) OpenEdition}

\section{Journals}

Édition électronique

URL : http://journals.openedition.org/edl/828

DOI : $10.4000 /$ edl. 828

ISSN : 2296-5084

Éditeur

Université de Lausanne

\section{Édition imprimée}

Date de publication : 15 mai 2015

Pagination : 101-114

ISBN : 978-2-940331-45-1

ISSN : 0014-2026

\section{Référence électronique}

Franc Schuerewegen, «Sur Jean Kaempfer, et sur Proust (à propos des pastiches) », Études de lettres [En ligne], 1-2 | 2015, mis en ligne le 15 mai 2018, consulté le 22 décembre 2020. URL : http:// journals.openedition.org/edl/828; DOI : https://doi.org/10.4000/edl.828 


\section{SUR JEAN KAEMPFER, ET SUR PROUST (À PROPOS DES PASTICHES)}

La structure du pastiche proustien est ternaire, non binaire. Cela veut dire que le pasticheur, quand il se livre à son exercice, imite toujours au moins deux textes à la fois. Le texte $\mathrm{X}$ le conduit au texte $\mathrm{Y}$ qui produit alors le texte $\mathrm{Z}$, à savoir le pastiche proprement dit. On peut dire d'une autre façon encore que les «belles phrases» sont toujours pour Proust des créations collectives et qu'il faut donc les appréhender comme telles. En passant, ces pages rendent hommage au grand universitaire qu'est le professeur Jean Kaempfer.

Entre les faits de la vie et le nom des hommes, il est de secrètes et d'inexplicables concordances. Kaempfer! Répétez-vous à vous-même ce nom composé de deux syllabes! Ne vous semble-t-il pas que vous voyiez le guerrier ne faisant qu'un avec son arme? Quoiqu'étrange et sauvage, ce nom a pourtant le droit d'aller à la postérité. Kaempfer! Toute la vie de l'homme est dans l'assemblage fantastique de ces huit lettres. Kaempfer! Dites cela et le bruit de la bataille remplit l'air. Kaempfer! Grand capitaine! Ce K qui précède Kaempfer offre à l'esprit je ne sais quoi de fatal. Kaempfer! Jean Kaempfer! "Jean" est proche de "génie». Je ne voudrais pas prendre sur moi d'affirmer que les noms et les hommes n'aient pas été créés en même temps par un Dieu réparateur. Peut-être reviendra-t-on quelque jour aux sciences occultes. Quel vent a soufflé sur ce nom magique, sur ce véritable sésame helvétique et planétaire? Notre globe est plein, tout s'y tient. Jean Kaempfer est né à La Chaux-de-Fonds, où il a fait ses classes. Chaudfontaine est une communauté francophone de Belgique. L'eau minérale de Chaudfontaine est exempte de nitrates. 
L'affaire Lemoine, la série de pastiches auxquels Proust a donné ce titre, commence "dans un roman de Balzac" ${ }^{1}$. Ce choix n'est évidemment pas anodin. L'ingénieur Lemoine, le protagoniste de "l'affaire», est aux yeux du pasticheur un personnage balzacien. Proust, en fait, a reconnu Lemoine sous les traits de Balthazar Claës dans La recherche de l'absolu. L'affiliation, pour qui a lu les textes, est facile à établir. On est à peu près au milieu du roman; Balthazar Claës est averti par son valet de chambre qu'une chose étonnante vient de se produire au laboratoire, où le savant ne va plus. Balzac écrit alors ceci:

- Monsieur, monsieur, criait Lemulquinier.

- Eh bien? dit Balthazar en se retournant.

- Un diamant!... ${ }^{2}$

C'est en effet "un diamant blanc de forme octaédrique» que le domestique tient à la main. Il l'a trouvé, explique-t-il, "dans la capsule qui communiquait avec cette pile que nous avions laissée en train de faire des siennes, et elle en a fait monsieur!». On sait la suite. Le maître des lieux est surpris et ému. Il a arrêté ses recherches trop tôt, il devra les poursuivre:

Hélas! si j’avais épié cette longue, cette lente, cette subite, je ne sais comment dire, cristallisation, transformation, enfin ce miracle, eh bien, mes enfants seraient bien plus riches encore ${ }^{3}$.

Balzac ne pense qu'à l'argent, la chose est connue. «La vulgarité de ses sentiments est si grande que la vie n'a pu l'élever ", c'est également Proust qui le dit ${ }^{4}$. Ce diamant surgi ex nibilo ou presque est donc tout d'abord pour le père de famille endetté un moyen de remettre ses finances à flot. Il refera sa fortune, ses enfants en profiteront. Mais on peut aussi lire le texte d'une autre manière. Claës est alors une figure de l'artiste et de l'écrivain. La «longue, lente, subite, je ne sais comment dire, cristallisation, transformation" qui a produit le diamant est une définition du style, plus exactement: elle définit la manière dont le style vient à l'écrivain. La scène de laboratoire, si on l'envisage sous cet angle, est un

I. M. Proust, L'affaire Lemoine, p. 8.

2. H. de Balzac, La recherche de l'absolu, p. 822.

3. Ibid., p. 823.

4. M. Proust, "[Sainte-Beuve et Balzac]», in Contre Sainte-Beuve, p. 263. 
art poétique. L'écrivain, donc, produit des phrases «belles comme des diamants".

Certes, je ne l'oublie pas, pour Proust, «dans Balzac, il n’y a pas à proprement parler de style». Chez lui "coexistent, non digérés, non encore transformés, tous les éléments d'un style à venir " 5 . Mais ces reproches faits à un confrère sont en eux-mêmes révélateurs. Proust, on le voit bien ici, envisage le style comme une transformation ou une transmutation de la matière verbale, bref: comme un phénomène chimique. La définition proustienne du style est balzacienne. Tout était déjà dans Balzac...

Où est alors Lemoine? Et nous ne perdons évidemment pas de vue que le héros de «l'affaire» est un escroc qui fait passer pour des diamants fabriqués dans son usine des pierres achetées chez des joailliers parisiens! L'escroc apparaît, chez Balzac, au détour d'une phrase. Je rappelle également la séquence. Quand le diamant est découvert, un sceptique prend la parole. Peut-on réellement avoir confiance en Balthazar Claës? Ce n'est pas si sûr:

Ce hasard est sans doute bien naturel, dit un de ces gens qui veulent expliquer tout, le bonhomme aura oublié quelque diamant véritable. C'est autant de sauvé sur ceux qu'il a brûlés ${ }^{6}$.

Proust a également vu ce passage et n'hésite pas à s'en servir. Supposons donc, comme on le suggère ici, que Claës ait fait une erreur de manipulation, admettons, en outre, puisque la chose est possible, que le chimiste ait confondu, même involontairement, un diamant naturel avec un diamant artificiel, à ce moment, il a agi comme Lemoine, et les deux personnages, Lemoine et Claës, n'en font plus qu'un seul. La recherche de l'absolu peut alors se lire comme une anticipation "balzacienne» d'une affaire "proustienne». Proust commence sa série de pastiches «dans Balzac» parce qu'il emprunte un sujet à Balzac. C'était tout simple.

\section{La pédale et le son}

J'ouvre maintenant "Journées de lecture», où Proust rend hommage au Capitaine Fracasse, livre chéri de son enfance:

5. Ibid., p. 269.

6. H. de Balzac, La recherche de l'absolu, p. 824. 
J'en aimais par-dessus tout deux ou trois phrases qui m'apparaissaient comme les plus originales et les plus belles de l'ouvrage. Je n'imaginais pas qu'un autre auteur en eût jamais écrit de comparables. Mais j’avais le sentiment que leur beauté correspondait à une réalité dont Théophile Gautier ne nous laissait entrevoir, une ou deux fois par volume, qu'un petit $\operatorname{coin}^{7}$.

Suit un bref pastiche du style de Gautier:

Le rire n'est point cruel de sa nature; il distingue l'homme de la bête, et il est, ainsi qu'il appert en l'Odyssée d'Homerus, poète grégeois, l'apanage des dieux immortels et bienheureux qui rient olympiennement tout leur saoul durant les loisirs de l'éternité.

Le pastiche est accompagné d'un commentaire sous forme d'aveu:

En réalité, cette phrase ne se trouve pas, au moins sous cette forme, dans Le capitaine Fracasse. Au lieu de "ainsi qu'il appert en l'Odyssée d'Homerus, poète grégeois», il y a simplement «suivant Homerus». Mais comme les expressions "il appert d'Homerus», "il appert de l'Odyssée», qui se trouvent ailleurs dans le même ouvrage, me donnaient un plaisir de même qualité, je me suis permis, pour que l'exemple fût plus frappant pour le lecteur, de fondre toutes ces beautés en une, aujourd'hui que je n'ai plus pour elles, à vrai dire, de respect religieux ${ }^{8}$.

On note la formule «fondre toutes ces beautés en une». Le pastiche est pensé comme un alliage des constituants. Balthazar Claës n'est pas loin. Mais si Claës est là, nous devons aussi accueillir son compagnon de route, ou son âme damnée, Lemoine. Chez Proust, les deux personnages marchent en effet de pair:

Et je pensais avec regret que l'exaltation avec laquelle je répétais la phrase du Capitaine Fracasse [...] aurait été plus délicieuse encore si j’avais pu trouver en une seule phrase de Gautier tant de ses charmes que mon propre artifice réunit aujourd'hui, sans parvenir, hélas! à me donner aucun plaisir ${ }^{9}$.

7. M. Proust, «Journées de lecture», p. 175.

8. Ibid.

9. Ibid., p. 176. 
On objectera ici que "Journées de lecture" est de 1904, alors que le premier pastiche où il est question de Lemoine, celui de Balzac, paraît dans Le Figaro en février 1908. Proust écrit sur Gautier bien avant que l'idée ne lui vienne d'entreprendre la série de 1908. Certes oui. Mais cela ne nous empêche pas d'affirmer, de façon rétrospective, qu'il y a un Claës et un Lemoine dans Proust pasticheur, et que Proust a sans doute consciemment choisi de se placer sous ce double parrainage.

Le pastiche est ce qui permet de produire, ou d'imiter, de manière «artificielle» ("que mon propre artifice réunit aujourd'hui») des richesses «naturelles», découvertes dans la sorte de gisement qu'est un livre aimé. En somme, le pastiche nait d'une forme de frustration, j'ajouterai: d'une gourmandise. Pour le lecteur admiratif, il n'y a jamais "assez» de belles phrases dans un texte; le pastiche est alors un moyen de lutter contre la pénurie. Mais cette sorte de continuation de l'œuvre par d'autres moyens («Il faut [...] laisser la pédale prolonger le son» ${ }^{10}$ ), si elle est jubilatoire, confond aussi les catégories du propre et de l'emprunté. Le pasticheur est alors à la fois Claës et Lemoine et il lui faut assumer cette double affiliation, ou identité.

Nous n'en sommes qu'au début de l'enquête; je passe à un deuxième temps où nous avons besoin de consulter le Journal des Goncourt, que je cite à la date du 26 avril 1893. Henry Standish, peintre anglais, prend la parole. Il est question de choses qui brûlent. A Balthazar Claës, on reprochait d'avoir "brûlé des diamants». Les Goncourt ont donc également ici un «côté Balzac»:

Et de cet incendie, il saute à un incendie aux environs de Londres, où sa femme ne se sauva qu'en sautant par la fenêtre, où une femme de chambre fut brûlée et où tout fut anéanti dans la maison, sauf un coffret de fer à bijoux qu'on retira du feu tout rouge. Les diamants étaient intacts, et un magnifique collier de perles était aussi intact, mais les perles étaient devenues toutes noires et, chose curieuse, toutes noires qu'elles étaient, avaient conservé leur orient ${ }^{11}$.

On retrouve la même anecdote dans le pastiche Goncourt du Temps retrouvé où n'apparaissent toutefois pas les diamants; il est alors seulement question de perles. Il faudra probablement en déduire que perles

IO. M. Proust, "A propos du "style" de Flaubert", p. 594.

II. J. de Goncourt, E. de Goncourt, Journal, p. 819. 
et diamants sont chez Proust des termes interchangeables, des sortes de synonymes, si l'on veut. Ces mots, et ces objets figurent emblématiquement dans le pastiche l'activité pastichante; objets "autotéliques", donc $^{12}$ :

Swann me fait admirer le collier de perles noires porté par la maîtresse de la maison et acheté par elle, toutes blanches, à la vente d'un descendant de Mme de La Fayette à qui elles auraient été données par Henriette d'Angleterre, perles devenues noires à la suite d'un incendie qui détruisit une partie de la maison que les Verdurin habitaient dans une rue dont je ne me rappelle plus le nom, incendie après lequel fut retrouvé le coffret où étaient ces perles, mais devenues entièrement noires ${ }^{13}$.

Standish est un citoyen britannique; chez Proust l'Angleterre revient dans le nom de la reine qui a offert le collier de perles à Mme de La Fayette. A Londres se trouve aussi le siège de la De Beers. Le pasticheur révèle donc ses «sources». Mais une autre leçon doit être retenue et ce n'est pas la moins importante. Proust écrit dans une note, à propos de L'affaire Lemoine:

Cette insignifiante affaire de police correctionnelle [...] fut choisie un soir par moi, tout à fait au hasard, comme thème unique de morceaux, où j'essaierais d'imiter la manière d'un certain nombre d'écrivains ${ }^{14}$.

On commence à fort bien comprendre que Proust n'a justement "pas choisi par hasard» le personnage de Lemoine comme héros des pastiches de 1908. Il a en somme, à ce moment, rencontré un double, comme il a trouvé un double en Balthazar Claës, comme celui-ci était - déjà un double pour l'auteur de La recherche de l'absolu. "Une escroquerie au diamant synthétique, pastiche de diamant, en somme: c'est le sujet de L'affaire Lemoine», signalait déjà, bien avant nous, Gérard Genette, dans «Flaubert par Proust» ${ }^{15}$. C'était aller tout de suite à l'essentiel.

I2. "Or je me suis permis plus qu'aucun de véritables débauches avec la délicieuse mauvaise musique qu'est le langage parlé, perlé, de Sainte-Beuve.» M. Proust, «A propos du "style" de Flaubert", p. 596.

I3. M. Proust, Le temps retrouvé, p. 293.

I4. M. Proust, "Dans un roman de Balzac», in L'affaire Lemoine, p. 7.

I5. G. Genette, «Flaubert par Proust», p. 35. 
Mais on peut être plus précis encore. Un second passage des Goncourt attire à présent notre attention. Je cite cette fois le Journal à la date du 15 août 1895 , où on lit ceci :

Puis comme il est question de son volume sur les pierres précieuses et que Daudet dit superstitieusement que la pierre précieuse est dangereuse, maléficiante, Montesquiou conte que lord Lytton, qui avait un culte pour la comtesse Greffulhe, lui avait laissé une pierre gravée admirable. Mais sur cette pierre gravée, il y avait des caractères qui intriguaient la comtesse. Elle la faisait porter à un mage, qui l'avertissait de se défaire au plus tôt de cette pierre sous peine de mort subite, ce qui était arrivé à Lytton. Là-dessus, la comtesse montait en voiture, se faisait conduire au bord de la Seine et jetait la pierre à l'eau: «C'est depuis ce temps, dit Montesquiou en riant, que le fleuve est si mauvais pour la santé parisienne» ${ }^{16}$.

Nous sommes une fois de plus en excellente compagnie. Apparaissent Montesquiou et la comtesse Greffulhe, "modèle" de la duchesse de Guermantes. Balzac, de son côté, que Montesquiou connaît fort bien - voir les pages de Sodome et Gomorrhe où Montesquiou-Charlus se révèle être un balzacologue hors pair ${ }^{17}$-, continue à veiller sur ce beau monde en coulisses. Le flamboyant aristocrate a à n'en pas douter pêché l'anecdote sur la pierre mystérieuse jetée dans la Seine dans La peau de chagrin, en l'arrangeant un peu, il est vrai. Et il y a plus. Le Journal annonce, de la plume de Montesquiou, un «volume sur les pierres précieuses». Ce volume ne peut être que Les perles rouges publié dans la Bibliothèque Charpentier en 1899. Proust connaît bien le texte de Montesquiou, qu'il cite à plusieurs reprises. Les perles ronges sont une évocation de Versailles et de ses jardins. Or qui dit Versailles dit Louis XIV, et qui dit Louis XIV dit... Saint-Simon. On voit le lien qui apparaît. Proust a aussi pastiché Saint-Simon, et le prétexte du pastiche est alors un portrait de Montesquiou. Il s'ensuit que Proust pastiche Saint-Simon à cause de, entre autres, Montesquiou:

I6. J. de Goncourt, E. de Goncourt, Journal, p. 1166.

17. M. Proust, Sodome et Gomorrhe, p. 442 sqq. 
Louis Quatorze est le seul Saint-Simon, / Le Grand Siècle écoulé survit en son grimoire / [...] Louis mène le char. Le Duc est au timon. / Les rayons du Soleil rentrent dans son armoire $[\ldots]^{18}$.

Poussons l'enquête plus loin, compliquons la donne. Il est aussi question, dans le pastiche de Saint-Simon, d'Yturri, le secrétaire de Montesquiou. Proust écrit alors ceci :

En un temps où chacun ne pousse guère ses vues plus loin qu'à faire distinguer son mérite, il avait celui [...] de mettre tout le sien à faire mieux éclater celui de ce comte [...] comme faisaient ces disciples qu'avaient accoutumé d'avoir toujours avec eux les anciens sophistes, ainsi qu'il appert des écrits d'Aristote et des discours de Platon ${ }^{19}$.

On relève le «ainsi qu'il appert». N'étions-nous pas chez Saint-Simon? Voilà qui nous ramène au Capitaine Fracasse et à Gautier! Une "perle» a été égarée, ou déplacée. Le pasticheur certes risque ici une sorte de practical joke, il s'amuse à brouiller les pistes. Mais s'il faut faire la part de l'humour, et de la provocation, c'est aussi, d'un autre point de vue, un secret d'atelier - de laboratoire - qui est ici révélé. On conçoit le plus souvent le pastiche comme une rencontre duelle, où le pasticheur est face au pastiché, le premier reproduisant la manière du second. Voir par exemple l'ouvrage de Paul Reboux et de Charles Muller, $A$ la manière de... (1908), qui a peut-être - on l'a dit - inspiré Proust. Est mis en place ici un dispositif plus complexe, et retors. Pour aller chez Saint-Simon, Proust choisit une série de détours passant notamment par Montesquiou et Gautier. L'art du pasticheur ne consiste pas à proprement parler à "imiter" la manière d'autrui, mais bien à "amalgamer" des formes et des styles, à les "fondre», comme le dit si bien "Journées de lecture», faisant le pari que, de cet amalgame, quelque chose de propre et, donc, de "proustien" pourra naître un jour.

Nous n'en avons pas fini avec Balzac, ni avec Montesquiou d'ailleurs, qui écrit dans la préface aux Perles rouges: «Balzac prétend que les noms agissent sur les individus, et réciproquement. ${ }^{20}$ Suit alors un exercice

I8. R. de Montesquiou, sonnet XLIII, "Le vrai maître», in Les perles rouges, p. 45.

19. M. Proust, "Dans les Mémoires de Saint-Simon", in L'affaire Lemoine, p. 49 sq.

20. R. de Montesquiou, Les perles rouges, p. XIII. 
de resémantisation, à la manière balzacienne, où deux noms de villes, Venise et Versailles, se voient couplés:

Quel échange de poésie et de mystère entre ces deux Cités fraternelles, Venise, par exemple, et Versailles, par ailleurs, et les syllabes qui les désignent! Venise, mot dans lequel il y a de la séduction de Vénus et du ton anisé des lagunes. Versailles, vocable où se mélangent la vibration du vers et celle du tressaillement ${ }^{21}$.

Proust retient l'allusion à Venise; il sait aussi que Montesquiou reprend un passage de $Z$. Marcas:

Entre les faits de la vie et le nom des hommes, il est de secrètes et d'inexplicables concordances [...]. Marcas s'appelait Zéphirin. Saint Zéphirin est très vénéré en Bretagne. Marcas était breton. / Examinez encore ce nom: Z. Marcas! Toute la vie de l'homme est dans l'assemblage fantastique de ces sept lettres [...]. Marcas! N'avez-vous pas l'idée de quelque chose de précieux qui se brise par une chute, avec ou sans bruit? ${ }^{22}$

On passe donc de Proust à Balzac via Montesquiou. La structure du pastiche proustien est ternaire, non binaire:

Bien plus, l'homme qui était alors à la tête de la plus colossale affaire de diamants de l'Angleterre s'appelait Werner, Julius Werner, Werner! ce nom ne vous semble-t-il pas évoquer bizarrement le moyen âge? Rien qu'à l'entendre, ne voyez-vous pas déjà le docteur Faust, penché sur ses creusets, avec ou sans Marguerite? N'implique-t-il pas l'idée de la pierre philosophale? Werner! Julius! Werner! Changez deux lettres et vous avez Werther. Werther est de Goethe ${ }^{23}$.

Le jeu de mots sur "Werner" et "Werther" doit sans doute beaucoup à celui qu'avait déjà risqué Montesquiou sur "Versailles» et "Venise». Proust, d'une certaine façon, prolonge la série. Il convient de saluer par ailleurs la présence de Faust, autre figure emblématique du pasticheur, après Claës et Lemoine. Marcas, je le signale en passant, rime avec Fracasse. Quant à Marguerite, faut-il rappeler que ce nom vient du latin

2I. Ibid., p. XIV.

22. H. de Balzac, Z. Marcas, p. 829 sq.

23. M. Proust, "Dans un roman de Balzac», in L'affaire Lemoine, p. 11 sq. 
margarita, signifiant "perle»? Examinez ce nom! On est chez Proust à mille lieues d'une simple esthétique de l'imitatio. Le pastiche est l'art de faire de l'art avec l'art. Le pastiche est l'autre nom de la littérature.

\section{Brève réflexion, pour finir, sur un blanc}

Une dernière question doit alors être soulevée; on verra qu'elle est, pour nous, dans le contexte qui est le nôtre, un peu inquiétante. Montesquiou, avons-nous dit, est chez Proust une référence cruciale, un intercesseur. Or il manque aux pastiches Lemoine un texte "à la manière de Montesquiou ». Comment expliquer cette lacune, ou absence? Je précise qu'il ne s'agit pas d'un oubli, Proust y a bel et bien songé. Il écrit au comte le 2 mars 1909: "Je suis trop fatigué, en ce moment, pour faire de nouveaux pastiches. Et le vôtre n'est même pas commencé.» ${ }^{24}$ Curieusement, il n’a jamais réalisé ce projet qui, pourtant, dans le cadre donné, s'imposait. Montesquiou salue Saint-Simon dans Les perles rouges. Proust fait le portrait de Montesquiou, à la façon de Saint-Simon, dans L'affaire Lemoine. Pourquoi n'y a-t-il pas, dans L'affaire Lemoine, un portrait de Saint-Simon à la manière de Montesquiou? Y aurait-il ici quelque chose comme une "case vide» chère aux poéticiens?

Proust répond lui-même à la question dans le pastiche de SaintSimon; il est toujours question de son ami aristocrate: «[...] il était aussi difficile de ne pas chercher à l'imiter que d'y parvenir.» ${ }^{25}$ Voilà qui est en réalité un autre aveu. Proust est à Montesquiou, aux idiosyncrasies de Montesquiou, ce que le chimiste, ou l'ingénieur-électricien sont au diamant, ce que l'amateur d'art est aux "perles». L'envie d'imiter est d'autant plus forte qu'il est à peu près sûr qu'elle ne pourra être assouvie. Montesquiou, en d'autres mots, est à la fois un modèle et un obstacle. Montesquiou, si l'on veut, est le nom d'une limite, ou de ce qui est ici présenté comme telle. Le désir mimétique est né chez Proust d'un objet s'appelant «Montesquiou». Or, pour le pasticheur, tragiquement en quelque sorte, cet objet demeure lui-même hors d'atteinte.

Certes, quelque chose de la voix de Montesquiou est passée dans celle de Charlus, et Proust imitait à merveille le maitre d'Yturri dans les

24. M. Proust, Correspondance, p. 54.

25. M. Proust, "Dans les Mémoires de Saint-Simon", in L'affaire Lemoine, p. 49. 
salons. Mais il ne s'agit pas ici d'amusements mondains; nous sommes dans l'antre du lion, c'est-à-dire dans le laboratoire du pasticheur. Nous sommes en d'autres mots au cœur de la littérature. On dit le plus souvent que Proust commence $A$ la recherche du temps perdu quand il renonce au Contre Sainte-Beuve, abandonnant l'idée d'un essai de théorie littéraire au profit d'une construction romanesque. Après les réflexions qui précèdent, une autre hypothèse peut être soutenue qui n'est sans doute pas, dans l'ordre d'idées qui est ici le nôtre, totalement absurde. Proust commence son roman au moment de se rendre compte qu'il n'est pas en mesure, alors qu'il brûle d'envie de le faire, de pasticher de façon crédible Montesquiou. Proust écrit la Recherche quand il réalise qu'il ne peut et ne doit être Montesquiou. Certes, au départ, il a pastiché involontairement l'œuvre du comte, comme d'autres l'avaient fait avant lui; il y a sans doute, par ailleurs, du pastiche involontaire dans les textes que nous a laissés Montesquiou. Mais un pastiche qui ne s'assume pas comme tel n'est pas un pastiche, et Proust est lui-même très bien placé pour le savoir. C'est bien pourquoi il décide de laisser incomplète la série sur Lemoine. Là où nous aurions en principe dû lire une imitation de la manière de Montesquiou, il y a donc un blanc, un vide. C'est ce vide qu'occupent aujourd'hui les deux mille pages de La recherche du temps perdu.

Montesquiou s'appelait Robert. Changez la première syllabe et vous avez Kaempfer. Kaempfer est au timon. Les rayons du soleil rentrent dans son armoire.

Franc SchuerewegeN

Université d'Anvers, Université Radboud de Nimègue 


\section{BIBLIOGRAPHIE}

Balzac, Honoré de, Z. Marcas, in La comédie humaine, éd. établie sous la direction de Pierre-Georges Castex, Paris, Gallimard, 1977, t. VIII, p. 829-854.

—, La recherche de l'absolu, in La comédie humaine, éd. établie sous la direction de Pierre-Georges Castex, Paris, Gallimard, 1979, t. X, p. 657-835.

Genette, Gérard, "Flaubert par Proust», in Gustave Flaubert, Paris, Editions Inculte, 2009, p. 31-60 (texte repris du numéro Flaubert de L'Arc, 79, 1980).

Goncourt, Jules de, Goncourt, Edmond de, Journal. Mémoires de la vie littéraire, t. III, texte intégral établi et annoté par Robert Ricatte, Paris, Robert Laffont, 2014.

Montesquiou, Robert de, Les perles rouges, Paris, Richard imprimeuréditeur, 1910.

Proust, Marcel, L'affaire Lemoine (1908 dans Le Figaro, repris dans Pastiches et mélanges, 1919), in Contre Sainte-Beuve, précédé de Pastiches et mélanges et suivi de Essais et articles, éd. établie par Pierre Clarac avec la collaboration d'Yves Sandre, Paris, Gallimard, 1971, p. 7-59.

—, "Journées de lecture» (1905), in Contre Sainte-Beuve, précédé de Pastiches et mélanges et suivi de Essais et articles, éd. établie par Pierre Clarac avec la collaboration d'Yves Sandre, Paris, Gallimard, 1971, p. 160-194.

-, Contre Sainte-Beuve, in Contre Sainte-Beuve, précédé de Pastiches et mélanges et suivi de Essais et articles, éd. établie par Pierre Clarac avec la collaboration d'Yves Sandre, Paris, Gallimard, 1971, p. 211-312.

—, "A propos du "style" de Flaubert" (1920), in Contre Sainte-Beuve, précédé de Pastiches et mélanges et suivi de Essais et articles, éd. établie par Pierre Clarac avec la collaboration d'Yves Sandre, Paris, Gallimard, 1971, p. 586-600. 
—, Correspondance, t. IX, éd. par Philippe Kolb, Paris, Plon, 1982 (1909).

-, Sodome et Gomorrhe (1922-1923), in A la recherche du temps perdu, éd. publiée sous la direction de Jean-Yves Tadié, 1988, t. III, p. $1-515$.

—, Le temps retrouvé (1927), in A la recherche du temps perdu, éd. publiée sous la direction de Jean-Yves Tadié, 1989, t. IV, p. 273-625. 
